\title{
Removal of Shadows and Reflections in the Images By Using Cross-Projection Tensors
}

\author{
B.HIMABINDU \\ (Asst. professor, Department of E.C.E, Chalapathi Institute of Technology, Guntur, A.P, India)
}

\begin{abstract}
We propose a new technique for removal of shadow and reflections in the images. We introduce cross projection tensors to achieve affine transformations of gradient fields. We used these tensors, to remove reflections in an image based on the edge of a second image information. Traditionally, the removal of the edges is achieved by setting the image gradients to zero on the basis of thresholds. One common application is in the Retinex problem, which recovers the illumination map the edges of the suppression of reflection, assuming that varies slowly.

We present a class of problems in which edge suppression can be a useful tool. The key idea of our approach is to obtain local tensors with an image and to transform the gradient field of another image with them. Reconstructed image of the modified gradient field shows suppressed edges or textures in the appropriate places. All operations are local and our approach does not require any global analysis.

We show that the algorithm in the context of applications such as: a) Elimination of shadows of color images and obtaining the illumination map b) Removing glass reflections.
\end{abstract}

Keywords: - Affine transformation, Cross projection tensors, Color image, Edge suppression, Gradient field.

\section{Introduction}

Our goal is the design of the disposal operations of shadows in images. A single threshold for the entire image cannot account for variations in lighting and reflection through the image. In this work, we propose a new method for the manipulation of the image field gradient based affine transformation with the tensioning of the projection. Our approach provides a way of removing the top edges of the image scene texture compared with the threshold. We make no assumptions about the ambient lighting, the smoothness of reflection or the illumination map and not using shadow masks explicit.

It shows how to eliminate shadows and reflections from glass in a picture, with a second image of the same scene taken under different lighting conditions. Our approach is based on obtaining the tensor projection of an image and uses it to transform the gradient field of another image. Presents techniques that work under natural conditions, as well as active illumination variations. For natural illumination, several images to calculate the intrinsic image and improve the estimation of illumination maps. For the active illumination, the flash is used in digital cameras attached to introduce additional lighting in the scene. These additional images are used to extract reliable information on the edges of the scene texture, avoiding hard thresholds and assumptions about the smoothness of the reflectance images or lighting.

\section{Objectives}

We present a class of problems in which the edge suppression can be a useful tool. Applications are displayed in the traditional problems such as recovery of the reflectance and demonstrate the utility of other problems such as the recovery of the reflection layer.

Intrinsic images are proposed as a useful description of the scene of the average level of Barrow and Tenenbaum [3]. The observed image is considered to be the product of reflectance image and an illumination image $[6,16]$.

Our approach can be used to eliminate complex scene structures such as layers of reflection due to glass. While photographing through the glass, flash photography usually have the unwanted reflections from objects in front of the glass. We show how to recover such layers of reflection. Agrawal et al. [1] proposed a gradient projection technique to remove reflections for the projection of the gradient of the flash intensity image on the ambient image intensity gradient. We show that the gradient projection algorithm is a special case of our approach, and presents color artifacts that can be eliminated by our method. Other methods for the elimination of reflection include change of polarization or the approach [9, 11] and Independent Component Analysis [5].

Local structure tensors and diffusion tensors derived from them were used for image processing and space-time optical flow [7], and regulation of PDE-based image [2, 12, 13, 15]. These approaches are based on the modification of image intensities by nonlinear diffusion equation 


$$
F_{t}=\operatorname{div}\left(D_{f} \nabla \mathrm{F}\right) \text {, }
$$

where div is the divergence operator, $\mathrm{\nabla F}$ is the gradient of the image and the $D_{f}$ represents the diffusion tensor. In comparison, our approach is a gradient based domain transformation of the gradient field $\nabla \mathrm{F}$ using $D_{f}$. Recently, gradient domain algorithms have been used for Poisson image editing [10], and the perfect image stitching [8].

\section{Affine Transformation on Gradient Fields}

Let $\mathrm{F}(\mathrm{x}, \mathrm{y})$ of image intensity and $\nabla \mathrm{F}=\left[\begin{array}{l}h_{x} \\ h_{y}\end{array}\right]$ denotes the gradient vector for I each pixel. The smoothed structure tensor $H_{\sigma}$ is defined as [12]

$$
H_{\sigma}=\left(\nabla \mathrm{FVF} F^{T}\right) * C_{\sigma}=\left[\begin{array}{cc}
h_{z}^{2} & h_{z} h_{y} \\
h_{z} h_{y} & h_{y}^{2}
\end{array}\right] * C_{\sigma}
$$

where $*$ denotes convolution and is a core $C_{\sigma}$ normalized 2D Gaussian of variance $\sigma$. The $H_{\sigma}$ matrix can be decomposed as

$$
H_{\sigma}=L \sum L^{T}=\left[\begin{array}{ll}
l_{1} & l_{2}
\end{array}\right]\left[\begin{array}{cc}
\lambda_{1} & 0 \\
0 & \lambda_{2}
\end{array}\right]\left[\begin{array}{l}
l_{1}^{T} \\
l_{2}^{T}
\end{array}\right]
$$

where $l_{1}, l_{2}$, denote the eigenvectors corresponding to eigen-values $\lambda_{1}$ and $\lambda_{2}$, respectively, and $\lambda_{2} \leq \lambda_{1}$. The eigenvalues and eigenvectors of $\mathrm{H}_{\sigma}$ to provide information about local structures in the image intensity [2]. For homogeneous regions, $\lambda_{1}=\lambda_{2}=0$. If $\lambda_{1}=0$ and $\lambda_{2}>0$, it means the presence of an edge intensity. The eigenvector $l_{l}$ corresponds to the direction of the edge.

Weickert $[14,15]$ proposed a generalization of the equation based on the divergence given by (1), where $D_{f}$ is a diffusion tensor field. At each pixel, $\mathrm{D}_{\mathrm{f}}(\mathrm{x}, \mathrm{y})$ is a $2 \times 2$ symmetric matrix, positive definite. Weickert proposed the design of the diffusion tensor $D_{f}$ for the selection of its eigen-vectors $m_{1}, m_{2}$ and eigen values of $\mu_{1}, \mu_{2}$ on the basis of eigen-values and eigen vectors of $\mathrm{G}_{\sigma} . D_{f}$ is then obtained as

$$
D_{f}=\left[\begin{array}{ll}
D_{f 11} & D_{f 12} \\
D_{f 21} & D_{f 22}
\end{array}\right]=\left[\begin{array}{ll}
m_{1} & m_{2}
\end{array}\right]\left[\begin{array}{cc}
\mu_{1} & 0 \\
0 & \mu_{2}
\end{array}\right]\left[\begin{array}{l}
m_{1}^{T} \\
m_{2}^{T}
\end{array}\right]
$$

Several designs for production of $\mathrm{D}$ have been proposed to coherence enhancing diffusion [2, 15], the edge enhancing diffusion [2], the restoration of the color image, in-painting, and magnification [13]. Usually, $\mathrm{D}_{\mathrm{f}}$ is obtained from the given image $F$. These approaches modify the image intensities using the diffusion equation (1). This article shows how to get the projection tensor and discuss the properties and applications of affine transformation of the field gradient $\nabla \mathrm{F}$ of the image with them.

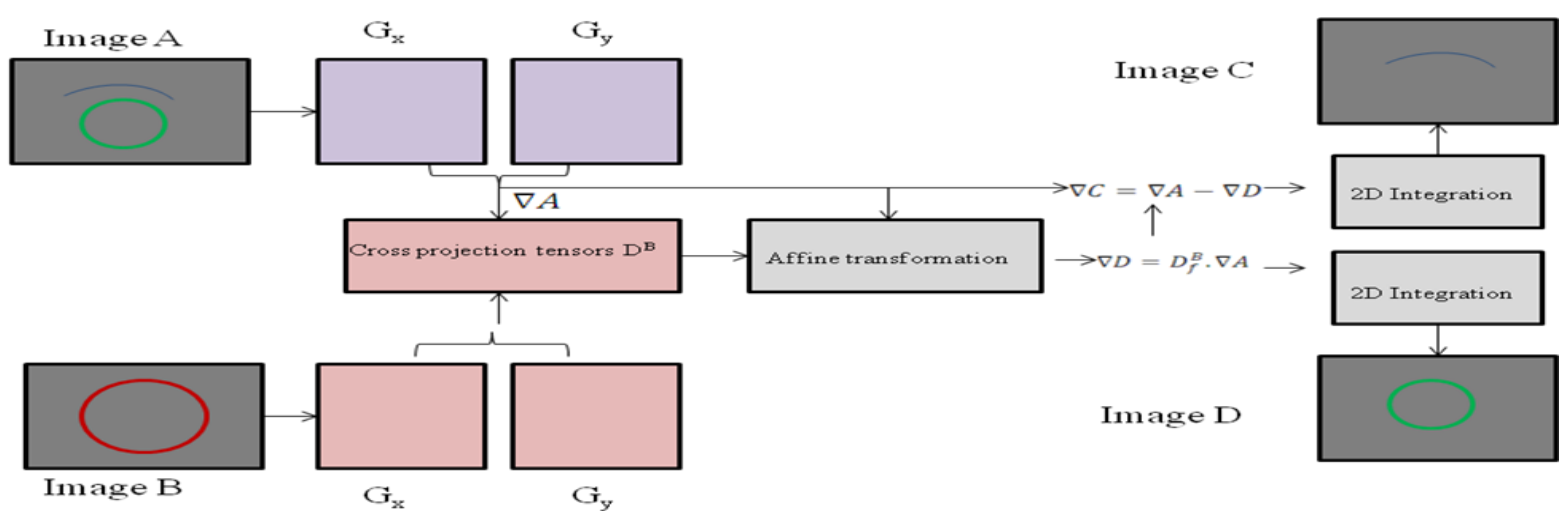

Fig. 1. Suppressing edges in image A using image B by affine transformation of gradient field using cross projection tensors. The cross projection tensor $\mathrm{D}^{\mathrm{B}}$ is obtained using images. The gradient field $\nabla \mathrm{A}$ is transformed using $\mathrm{D}^{\mathrm{B}}$ to give $V \mathrm{D}$, removing those edges from $\mathrm{A}$ which are present in $\mathrm{B}$. Reconstruction from $\nabla \mathrm{D}$ gives image $\mathrm{D}$, with corresponding edges suppressed. Reconstruction from the difference gradient field $(\nabla \mathrm{A}-\mathbb{\nabla})$ gives image $\mathrm{C}$, which preserves those edges in $\mathrm{A}$ which are also present in $\mathrm{B}$. 


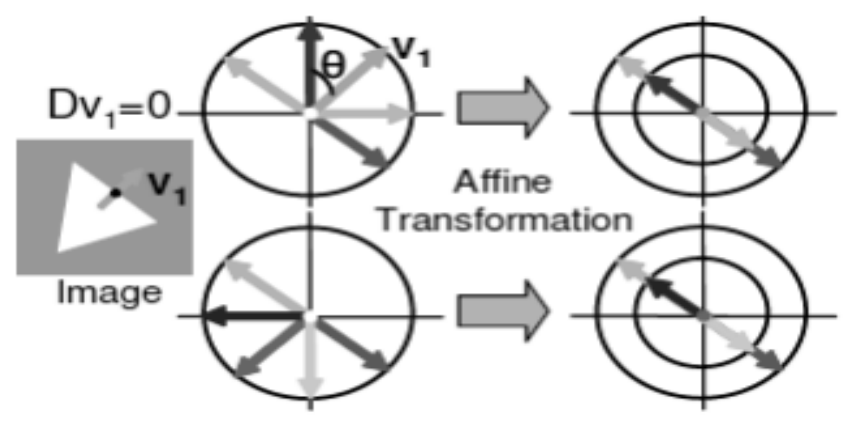

Fig. 2. Visualizing affine transformation on gradient vectors. At each pixel in an image, $l_{l}$ corresponds to the direction of the dominant edge. After affine transformation using $D_{\mathscr{S}}^{\mathrm{m} Z \mathrm{~V}}$, any vector gets projected to the direction orthogonal to the local gradient vector $l_{l}$.

\subsection{Gradient Projection}

The technique of gradient projection (GP) to remove artifacts from the flash image using an image without flash environment. They project the flash image gradient in the direction of the ambient image gradient to remove spurious edges from flash image due to glass reflections due to reflections from glass. Using the idea that the direction of the gradient image is stable to changes in illumination [4]. First, show that taking a projection may also be defined by an affine transformation of the gradient field.

The eigen-vector $l_{l}$ of the structure tensor matrix $\mathrm{G}_{\sigma}$ corresponding to the edge direction. Suppose we define the self-projection $D_{f}^{g e l f}$ tensor as

$$
\begin{aligned}
& m_{1}=l_{1} s \quad m_{2}=l_{2}, \quad \mu_{1}=0 \mu_{2}=1_{s} \\
& D_{f}^{g E l f}=\left[\begin{array}{ll}
l_{1} & l_{2}
\end{array}\right]\left[\begin{array}{ll}
0 & 0 \\
0 & 1
\end{array}\right]\left[\begin{array}{l}
L_{1}^{T} \\
l_{2}^{T}
\end{array}\right] \quad \text { (4) }
\end{aligned}
$$

It is easy to see that an affine transformation of the image Gradient using $D_{f}^{g s l f}$ will remove the local edge.

$$
\begin{aligned}
D_{f}^{\text {gElf }} \mathbb{l}_{1} & =\left[\begin{array}{ll}
\mathbb{l}_{1} & \mathbb{l}_{2}
\end{array}\right]\left[\begin{array}{ll}
0 & 0 \\
0 & 1
\end{array}\right]\left[\begin{array}{l}
\mathbb{L}_{1}^{T} \\
\mathbb{l}_{2}^{T}
\end{array}\right] \mathbb{l}_{1} \\
& =\left[\begin{array}{ll}
\mathbb{R}_{1} & \mathbb{l}_{2}
\end{array}\right]\left[\begin{array}{ll}
0 & 0 \\
0 & 1
\end{array}\right]\left[\begin{array}{l}
1 \\
0
\end{array}\right]=\left[\begin{array}{l}
0 \\
0
\end{array}\right]
\end{aligned}
$$

Fig.2 shows the effect of the gradient vector transformation using $D_{f}^{\text {self }}$ other. All vectors are projected to the direction orthogonal to the local gradient vector $l_{1}$. Thus, we can establish the following relationship. The transformation of a vector with $D_{f}{ }^{\text {self }}$ is equivalent to the projection in the orthogonal direction of the local gradient vector

The gradient projection approach as described in [1] cannot handle homogeneous regions and introduces color artifacts. This is because it does not include support for the estimation of gradient direction neighborhood, which is unstable in the presence of noise and low frequency regions. Furthermore, the projection is done for each channel separately which leads to color artifacts. Our approach combines spatial data (with $\sigma>$ 0 ) and in all channels to handle the noise and have no color artifacts.

\section{Cross-Projection Tensors}

We now show how to remove the scene texture edges of an image by transforming the gradient field using cross-projection tensors obtained from a second image of the same scene (see Fig. 1). The final image is obtained by a $2 \mathrm{D}$ integration of the modified gradient field.

Let $\mathrm{A}$ and $\mathrm{B}$ represent the two images. That $H_{\sigma}^{A}$ and $H_{\sigma}^{E}$ denote the smoothed structure tensors for image of A and B, respectively. The eigen values and eigen-vectors of the $H_{\sigma}^{A}$ and $H_{\sigma}^{B}$ is denoted by superscripts $\mathrm{A}$ and $\mathrm{B}$, respectively. The technique for obtaining the cross projection tensor $D_{f}^{B}$ is now explained. Note that by transforming $\nabla \mathrm{A}$ with $D_{f}^{B}$, we (a) removing all edges of the A that are present in $\mathrm{B}$, and (b) retain all edges in $\mathrm{A}$ which are not in B. For $D_{f}^{B}$, we propose the following rules: 
- $m_{1}=l_{1}^{B}, m_{2}=l_{2}^{B}$

- If $\mathrm{B}$ is homogeneous $\left(\lambda_{1}^{B}=0\right)$

- If A is also homogeneous $\left(\lambda_{1}^{A}=0\right)_{s}$ set $\mu_{1}=\mu_{2}=0$. This results in $D_{f}\left(x_{v} y\right)=\left[\begin{array}{ll}0 & 0 \\ 0 & 0\end{array}\right]$ for that pixel.

- If A is not homogeneous $\left(\lambda_{1}^{A}>0\right)_{s}$ set $\mu_{1}=\mu_{2}=1$. This results in $D_{f}\left(x_{v} y\right)=\left[\begin{array}{ll}1 & 0 \\ 0 & 1\end{array}\right]$ and edges which are in $\mathrm{A}$ but not in $\mathrm{B}$ can be retained.

- $\quad$ Else, if there is an edge in $B\left(\lambda_{1}^{B}>0\right)$, remove that edge by setting $\mu_{1}=0, \mu_{2}=1$.

In practice, due to noise and the estimation of the gradient using finite differences, a small non-zero value is used as a threshold to decide for homogeneity. In homogeneity threshold needs to vary across the image, if the image has spatially varying illumination. Since taking into account the direction of the edge, not necessary spatially adaptive thresholds.

\subsection{Combining information across color channels}

The above formulation can be used for grayscale images. A naive way of handling color images would be to get the cross projection tensor of each channel and transform the gradient field in each channel separately. However, this scheme introduces color artifacts in the reconstructed image as the projection tensor does not use the information in all channels. We obtain a common cross projection tensor for all channels by estimating a common Ho matrix as [12]

$$
\left.H_{\sigma}=\sum_{i=1}^{a}\left(\nabla F_{i} \nabla F_{i}^{T}\right)\right) * C_{\sigma}
$$

\subsection{Removing shadows from color images}

\section{Results for Applications}

We use a flash image B of the scene to remove shadows from the ambient (no-flash) image A. The flash and ambient images were captured in rapid succession with the usefulness of remote capture with the camera on a tripod. We obtain the cross projection tensor $D_{f}^{B}$ using B and transform the gradient field $\nabla \mathrm{A}$ using it. The recovered shadow free image $\mathrm{C}$ has no color artifacts and the recovered illumination map $\mathrm{D}$ is free of strong texture edges. Fig. 3 shows a challenging scenario where the hat on the mannequin casts shadows on the mannequin's face and neck. Generally, the ambient and flash images have a different color tone due to the ambient light is yellow-red and flash illumination is blue. Our algorithm not calibration requires pre-processing or color calibration and no color artifacts compared with the result using gradient projection. One might think that the ratio image $\mathrm{A} / \mathrm{B}$ could give the illumination map of the scene. However, the ratio image does not represent the illumination map due to the effects of flash shadows and variations in illumination due to the flash. The illumination map obtained by our approach better represents the diffuse ambient illumination.

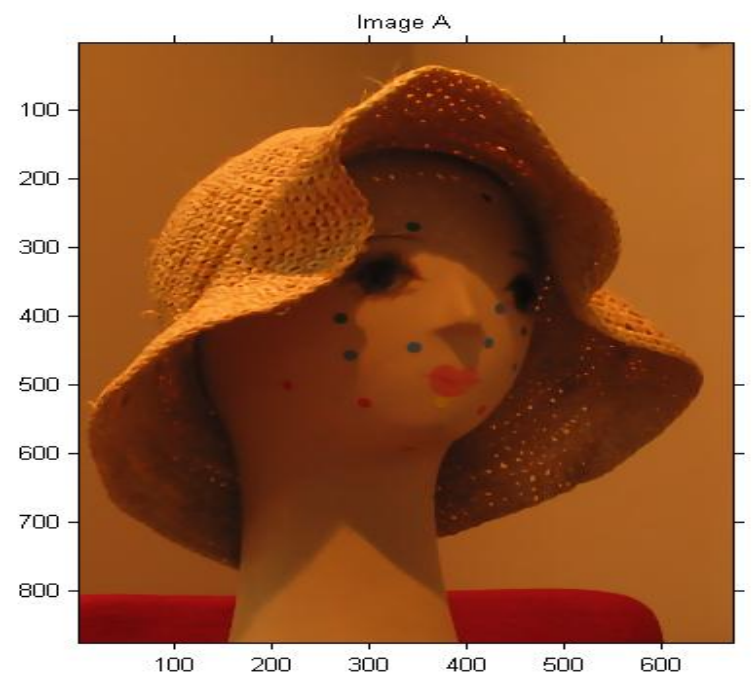

(a)

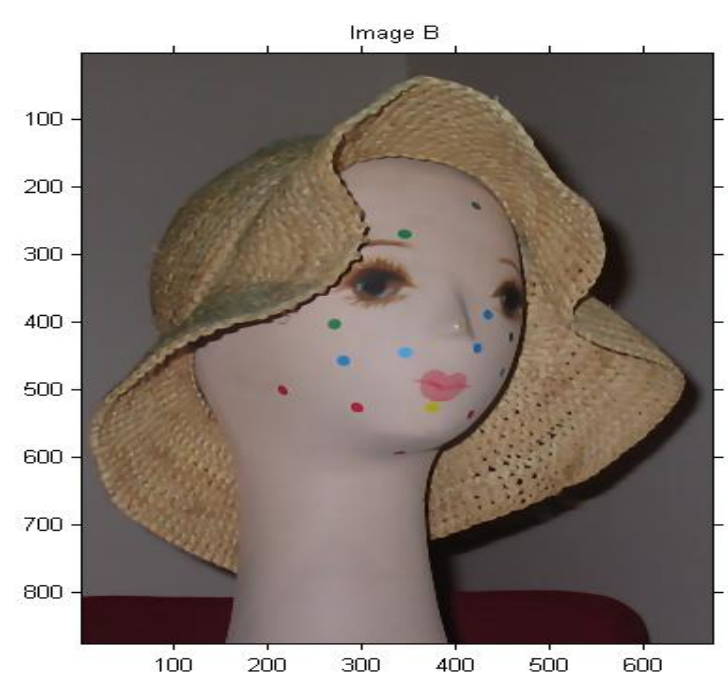

(b) 


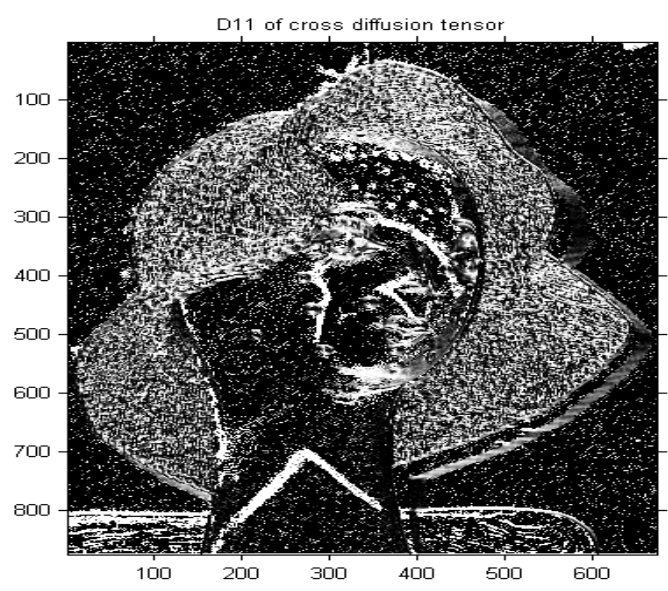

(c)

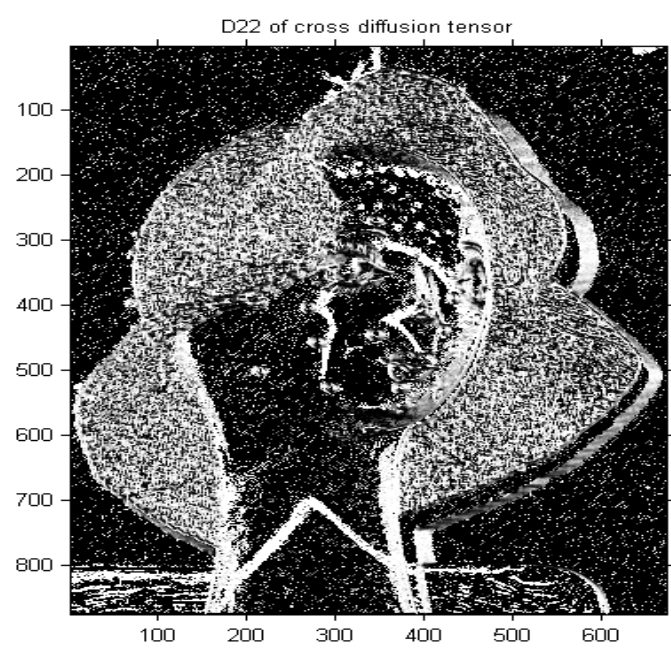

(e)

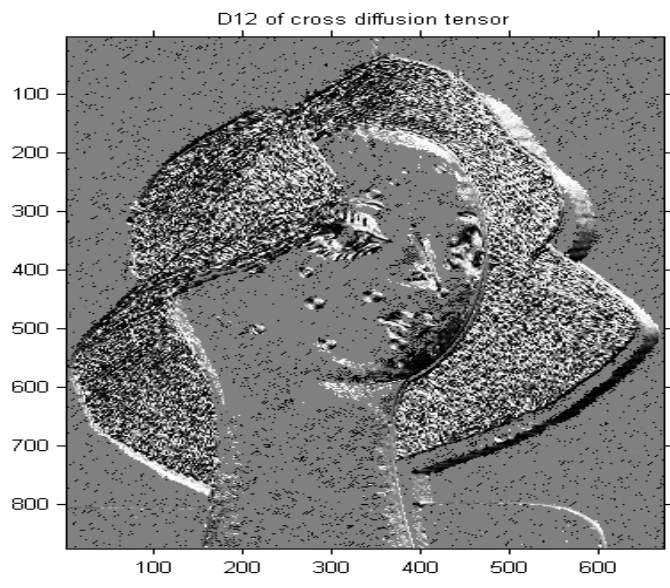

(d)

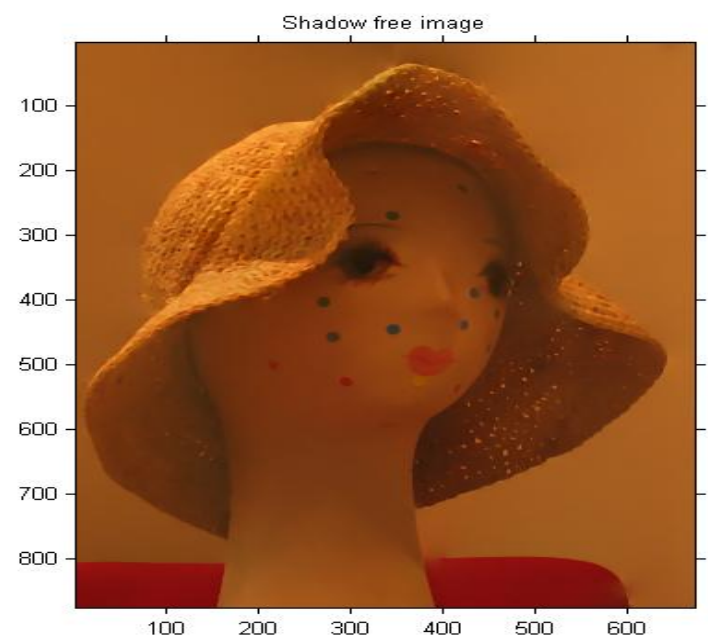

(f)

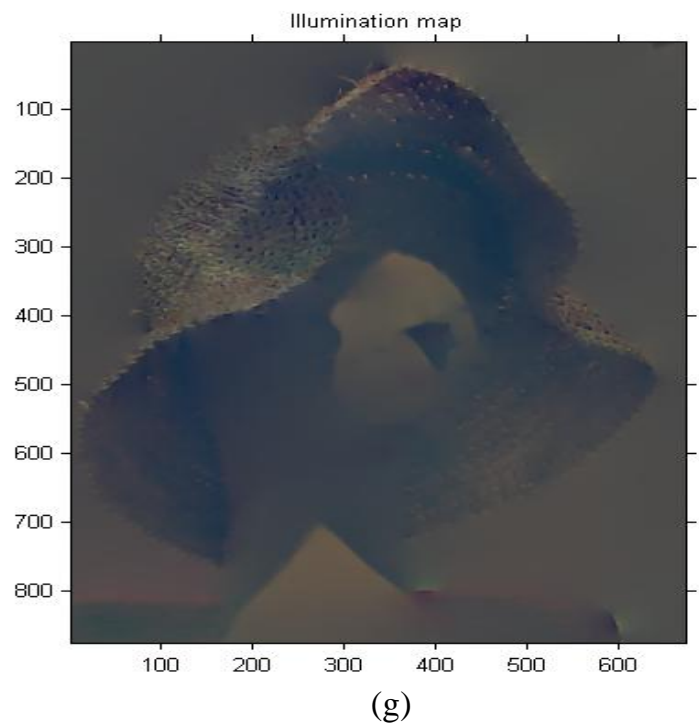

Fig:3 (a) Ambient image A of a mannequin. The hat casts shadows on the mannequin's face and neck in the ambient image A. (b) flash image B of a mannequin. The flash image B is taken with a short exposure time. (c) image with component $\mathrm{D}_{11}$ of cross projection tensor. (d) image with component $\mathrm{D}_{12}$ of cross projection tensor. (e) image with component $\mathrm{D}_{22}$ of cross projection tensor. (f) Shadow free image C. (g) illumination Image D. 
Removal Of Shadows And Reflections In The Images By Using Cross-Projection Tensors

\subsection{Removing glass reflections}

While photographing through glass in low light, an ambient image is usually of low quality and has low contrast. Using a flash improves contrast, but can result in reflections of objects in front of the glass. Fig. 4 shows an example where the camera is looking at an office scene through a glass window. The image has unwanted reflections flash chess board out of the window glass. We use the ambient image B to obtain the cross projection tensor $D_{f}^{B}$ and transform the gradient field $\nabla A$ of flash image $\mathrm{A}$ in use. The reflective layer is obtained by integrating the image $\nabla \mathrm{C}$ and free flash reflection is obtained by integrating $\nabla A-\nabla C$. The affine transformation is repeated 5 times as the reflection layer has strong edges. In comparison, one can see a light reflective stain remaining on the result of gradient projection.

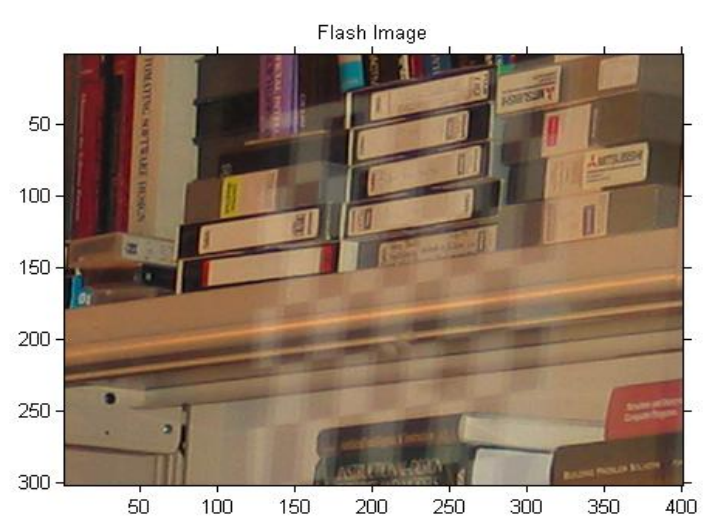

(a)

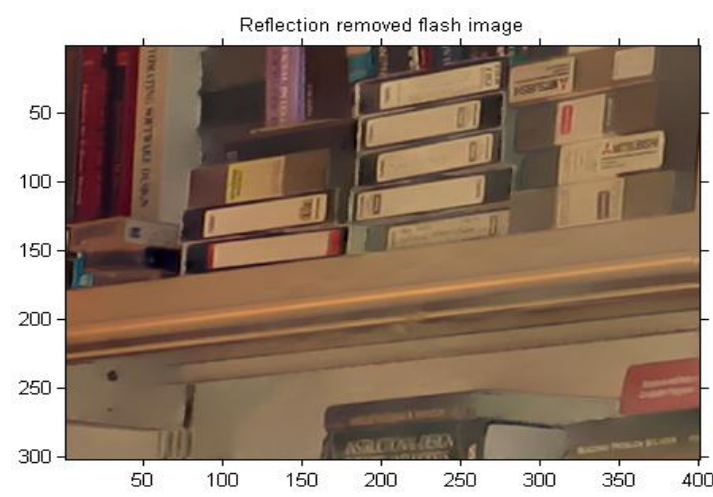

(c)

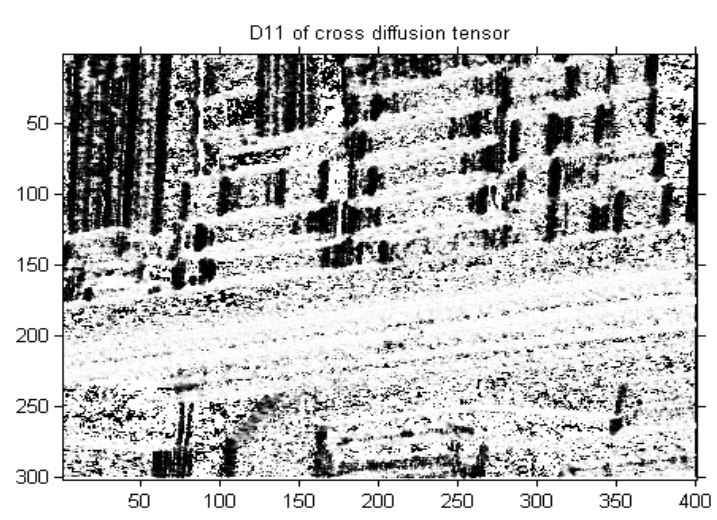

(e)

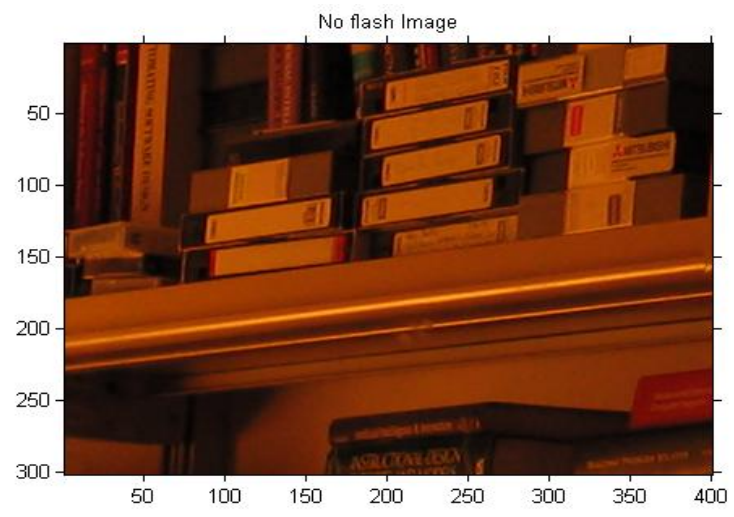

(b)

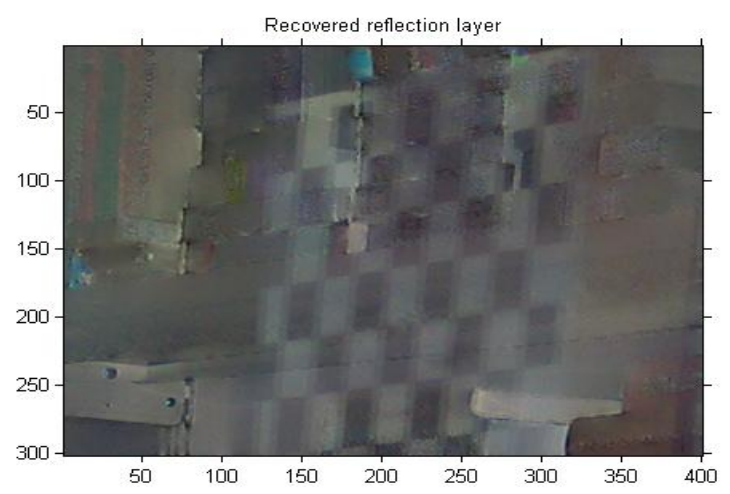

(d)

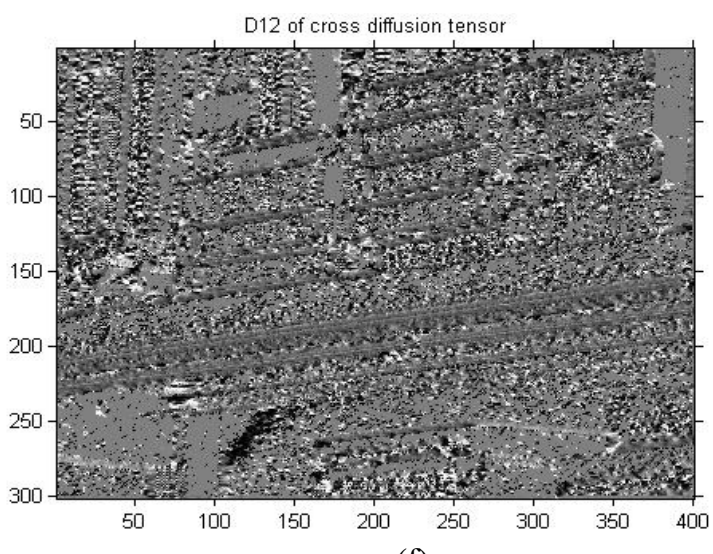

(f) 


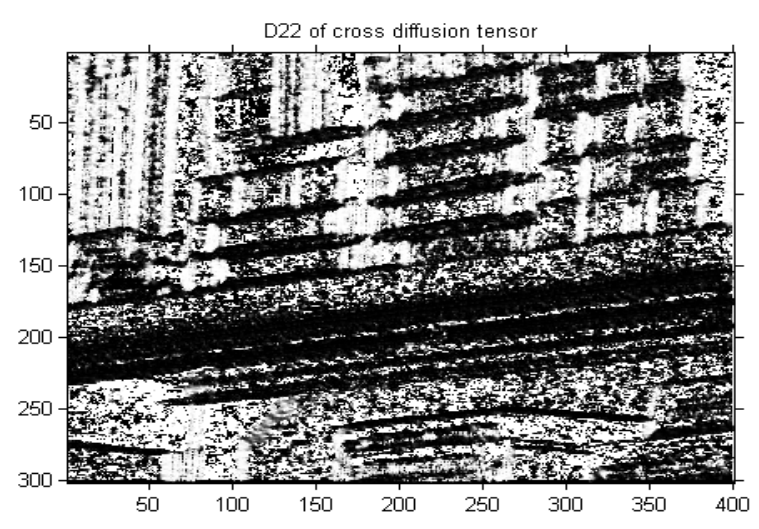

(g)

Fig.4. (a) Ambient Image A. (b) Image without flash B. (c) Reflection removed flash image C. (d) Recovered flash layer Image D. (e) image with component $D_{11}$ of cross projection tensor. (f) image with component $\mathrm{D}_{12}$ of cross projection tensor. $(\mathrm{g})$ image with component $\mathrm{D}_{22}$ of cross projection tensor.

\section{Conclusion}

We have made a proposal for edge suppressing operations on an image, based on the affine transformation of gradient fields using cross projection tensor derived from another image. Our focus is local and does not require a global analysis. In recovering the illumination map, we do the usual assumption that the edges of the scene texture not coincide with the edges of illumination. This can be handled by incorporating global information on the design of cross projection tensors. We used a variation of $\sigma$ to estimate the structure tensor $\mathrm{G}_{\sigma}$, but an adaptive neighborhood scheme could improve the results. We showed applications on shadows and glass reflections from images, recovering the illumination map, and estimating intrinsic images in nonLambertian scenes. Our approach is conceptually simple and can easily handle color images without any color calibration. We are optimistic that this framework can be used for handling edge beyond suppression and edge preservation and for applications in image interpretation, editing and special effects.

\section{References}

[1] A. Agrawal, R. Raskar, S. Nayar, and Y. Li. Removing photography artifacts using gradient projection and flashexposure sampling. ACM Trans. Graph., 24(3):828-835, 2005.

[2] G. Aubert and P. Kornprobst. Mathematical Problems in Image Processing: Partial Differential Equations and the Calculus of Variations, volume 147 of Applied Mathematical Sciences. Springer-Verlag, 2002.

[3] H. Barrow and J. Tenenbaum. Recovering intrinsic scene characteristics from images. In Computer Vision Systems, pages 3-26, 1978.

[4] H. Chen, P. Belhumeur, and D. Jacobs. In search of illumination invariants. In Proc. Conf. Computer Vision and Pattern Recognition, pages $254-261,2000$.

[5] H. Farid and E. Adelson. Separating reflections and lighting using independent components analysis. In Proc. Conf. Computer Vision and Pattern Recognition, pages 1262- 1267, June 1999.

[6] B. Horn. Determining lightness from an image. Comput. Graphics, Image Processing, 3:277-299, 1974.

[7] B. Jahne. Spatio-Temporal Image Processing, Theory and Scientific Applications, volume 751 of Lecture Notes in Computer Vision. Springer-Verlag, Berlin, Germany, 1993.

[8] A. Levin, A. Zomet, S. Peleg, and Y.Weiss. Seamless image stitching in the gradient domain. In ECCV (4), pages 377-389, 2004.

[9] S. Nayar, X. Fang, and T. Boult. Separation of reflection components using color and polarization. Int'l J. Computer Vision, 21(3):163-186, Feb. 1997.

[10] P. Perez, M. Gangnet, and A. Blake. Poisson image editing. ACM Trans. Graph., 22(3):313-318, 2003.

[11] Y. Schechner, N. Kiryati, and R. Basri. Separation of transparent layers using focus. Int'1 J. Computer Vision,39(1):25-39, Aug. 2000.

[12] D. Tschumperle. PDE's Based Regularization of Multivalued Images and Applications. PhD thesis, Universite de Nice - Sophia Antipolis, dec 2002.

[13] D. Tschumperle and R. Deriche. Vector-valued image regularization with pdes : A common framework for different applications. In Proc. Conf. Computer Vision and Pattern Recognition, volume 1, 2003.

[14] J. Weickert. A review of nonlinear diffusion filtering. In Scale-Space Theory in Computer Vision, Lecture Notes in Comp. Science (Springer, Berlin), 1997.

[15] J. Weickert. Coherence-enhancing diffusion filtering. Int'1 J. Computer Vision, 31:111-127, 1999.

[16] Y. Weiss. Deriving intrinsic images from image sequences. In ICCV, pages 68-75, 2001. 